\title{
Analysis methodology based on temporal FEM for bio-mechanical simulations
}

\author{
A. Eriksson \\ KTH Mechanics, Royal Institute of Technology, Stockholm, Sweden
}

\begin{abstract}
This paper deals with the analysis of optimal path forward dynamics of mechanical systems. The primary applications considered are bio-mechanical simulations of musculoskeletal systems. The focus is put on problems of controlled movements from one configuration to another. Different criteria for this movement are allowed in the formulation. Formulations, examples and conclusions are given.
\end{abstract}

\section{Introduction}

This paper deals with the analysis of optimal path forward dynamics of mechanical systems. The primary applications considered are bio-mechanical simulations of musculoskeletal systems. Controlled problems are considered, with an initial state, but also a limited number of target states at certain time instances. An optimal path is sought, which produces the movement at the lowest possible cost. Different choices of cost functions are considered.

The methodology uses a temporal finite element formulation, based on Hermitian interpolation of all displacement coordinates, [1]. The problem is using a limited number of displacement coordinates for kinematic description. The basic principle is a time instance set of dynamic equilibrium equations, linear or nonlinear, involving the external loads, and unknown control forces to be decided. The control forces can be forces in certain coordinates at certain time stations, typically joint moments, but the setting allows muscular forces, or even the muscular nominal stresses to be used as unknowns.

The main assumption and limitation in the present simulations, when seen in the musculoskeletal context, is that the forces can be infinitely quickly regulated, without delays and not considering the activation dynamics. Restrictions on displacement coordinates or controls can be introduced. Redundancy in the force system 
causes no additional problems. As a post-processing of results, unlimited force resultants can give muscular forces which fulfil limits in possible values, but do not fulfil formal global optimality.

Alternative methods for solution of the controlled problem have been discussed and evaluated in $[1,2]$. These are based on standard methods for time integration of an evolution problem, [3]. It is, however, suggested that the proposed methodology has certain advantages, especially for problems with low to moderate numbers of degrees of freedom.

The optimality of movement is a much debated issue. In the present work, it is assumed that the optimality is measured by some cost function; specific choices are easily introduced in the algorithm. An interesting alternative to the minimization of needed forces is to emphasize the physiological demand for smooth motion patterns, by basing an optimality on jerk measures. Variations of the minimum-jerk model formulated by Flash and Hogan, [4], have been used both in the diagnosis of pathology, [5], and as a tool for evaluating the kinematics of both human and robotic or prosthetic movement, [6-8]. The inclusion of a jerk measure is briefly discussed in the paper. Whether any one criterion is valid for all kinds of movements and in all situations is not discussed, [9].

\section{Basic formulation}

The main formulation of the problem has been given in [10], but is here repeated in condensed form.

\subsection{Time instance equilibrium equations}

A simple static solution often provides essential information about the main characteristics of a structural system, but may not be sufficient for the understanding of large inertia effects, or for time-dependent loads. The dynamic formulation can be stated in several ways, dependent on application, [11]. The present discussion handles both linear and non-linear structural formulations, and allows arbitrary time variations of the loads. It further allows large displacements, and load positions dependent on these displacements; the load cases must be conservative. The formulation considers a priori unknown control forces on the system, chosen to fulfil demands on certain target displacement states, often with an optimality postulate.

The dynamic problem is thus governed by a time instance discrete dynamic equilibrium equation of the form, [2]:

$$
\mathbf{M a}(t)+\boldsymbol{f}(\boldsymbol{u}(t), \boldsymbol{v}(t))-\boldsymbol{p}(t)-\mathbf{E}_{c} \boldsymbol{c}(t)=\mathbf{0}
$$

using the collected displacements $\boldsymbol{u}(t)$, velocities $\boldsymbol{v}(t)$ and accelerations $\boldsymbol{a}(t)$ in $N_{d}$ displacement coordinates. Displacement independent external forces are prescribed as $\boldsymbol{p}(t)$, whereas $\boldsymbol{f}$ describes all internal forces and displacement affected loads. The effects of control forces $c(t)$ are described by an action description 
matrix $\mathbf{E}_{c}$, of size $N_{d} \times N_{c}$. In order to allow a relevant cost function for control, the matrix must be correctly scaled. With these assumptions, a time instance residual form is written:

$$
\boldsymbol{e}(t) \equiv \boldsymbol{e}(\boldsymbol{u}(t), \boldsymbol{v}(t), \boldsymbol{a}(t), \boldsymbol{p}(t), \boldsymbol{c}(t) ; t)=\mathbf{0}
$$

\subsection{Interpolation of displacements}

The formulation uses a discretized description in the time dimension, using a predefined set of $N_{t}+1$ time stations within the time interval $0 \leq t \leq T$. At time station $t^{j}=\frac{j}{N_{t}} T, \quad\left(j=0, \ldots, N_{t}\right)$, the current configuration of a system is described by $N_{d}$ displacement coordinates $q_{i}^{j}\left(1 \leq i \leq N_{d}\right)$, and their time differentials $\dot{q}_{i}^{j}$. Values and differentials of coordinate $i$ at all the time stations are collected in a vector:

$$
\boldsymbol{Q}_{i}=\left[q_{i}^{0}, \dot{q}_{i}^{0}, q_{i}^{1}, \ldots, \dot{q}_{i}^{N_{t}}\right]^{\mathrm{T}}
$$

The displacements are interpolated in the time variable, from the displacement coordinates:

$$
u_{i}(t)=[\mathbf{N}(t)] \boldsymbol{Q}_{i}
$$

where the functions in the matrix $[\mathbf{N}(t)]$ describe the time interpolation, [10]. Only a few functions are non-zero at time $t,[12]$. A local Hermitian form gives $C^{1}$ time continuity over time element borders, [13].

With the same time interpolation applied to all components, the displacements at time $t$ can be written:

$$
\boldsymbol{u}(t) \equiv\left[u_{1}(t), \ldots, u_{N_{d}}(t)\right]^{\mathrm{T}}=[\mathbb{N}]\left[\mathbf{Q}_{1}^{\mathrm{T}}, \mathbf{Q}_{2}^{\mathrm{T}}, \ldots, \mathbf{Q}_{N_{d}}^{\mathrm{T}}\right]^{\mathrm{T}} \equiv[\mathbb{N}] \boldsymbol{Q}
$$

with a sparse matrix $[\mathbb{N}]$ of size $\left(N_{d}\right) \times 2 N_{d}\left(N_{t}+1\right)$. Similarly, velocity and acceleration components are:

$$
\begin{aligned}
& \boldsymbol{v}(t)=[\dot{\mathbb{N}}] \boldsymbol{Q} \\
& \boldsymbol{a}(t)=[\ddot{\mathbb{N}}] \boldsymbol{Q}
\end{aligned}
$$

The jerk components, i.e., the third time differential of the displacements, are:

$$
\boldsymbol{j}(t)=[\dddot{\mathbb{N}}] \boldsymbol{Q}
$$

and are constants in each interval, due to the Hermitian functions. 


\subsection{Control forces}

The $N_{c}$ control force components $c_{i}(t)$ are linearly time-interpolated from their values at $N_{k}$ chosen time stations. For each component, the discrete representation is:

$$
\boldsymbol{C}_{i}=\left[c_{i}\left(t^{c 1}\right), c_{i}\left(t^{c 2}\right), \ldots, c_{i}\left(t^{c N_{k}}\right)\right]^{\mathrm{T}}
$$

with $t^{c 1} \geq 0$ and $t^{c N_{k}} \leq T$, formally independent of the time stations for the displacement coordinate discretization. The full control force variation is discretized by:

$$
\boldsymbol{C}=\left[\mathbf{C}_{1}^{\mathrm{T}}, \mathbf{C}_{2}^{\mathrm{T}}, \ldots, \mathbf{C}_{N_{c}}^{\mathrm{T}}\right]^{\mathrm{T}}
$$

and interpolated to a specific time instance as:

$$
\boldsymbol{c}(t) \equiv\left[c_{1}(t), \ldots, c_{N_{c}}(t)\right]^{\mathrm{T}}=\left[\mathbb{N}_{c}\right] \boldsymbol{C}
$$

where the matrix $\left[\mathbb{N}_{c}\right]$ is $N_{c} \times\left(N_{c} N_{k}\right)$, but very sparse.

\subsection{Algebraic equations}

The time instance form given by Eq. (2) is used in different ways to state the full problem. With either approach, a set of $2 N_{d} N_{t}$ equations is formulated:

$$
\boldsymbol{E}(\boldsymbol{Q}, \boldsymbol{C}, \boldsymbol{P})=\mathbf{0}
$$

with $\boldsymbol{P}$ a consistent representation of the external forces $\boldsymbol{p}(t)$ for all time stations. In a point collocation, [10], the equations are formulated simultaneously for two points within each of the $N_{t}$ time intervals.

\subsection{Dynamic feasibility: restrictions and limits}

A set of $N_{b}$ linear conditions on the discrete coordinates are introduced by:

$$
\boldsymbol{B}(\boldsymbol{Q}) \equiv \mathbf{B}_{q} \boldsymbol{Q}-\boldsymbol{b}_{q}=\mathbf{0}
$$

At least $2 N_{d}$ conditions are needed to define an initial state at $t=0$. Excessive boundary conditions define a target state, and imply the need for at least $N_{c} N_{k}=$ $N_{b}-2 N_{d}$ free control force components. The interpolation allows inclusion of both velocity and acceleration conditions in the same form.

During the dynamic process, mechanical or physiological restrictions on controls or movements define a feasibility region for the mathematical optimization. The restrictions are valid at all time stations, thus making the number of potentially active restrictions high. They can often be seen as linear inequalities in the coordinates, e.g.:

$$
\mathbf{B}_{R} \boldsymbol{Q}-\boldsymbol{b}_{R} \geq \mathbf{0}
$$

The non-linearity in the problem, however, leads to an iterative choice of active 
restrictions. At an iteration stage, these introduce equality in a subset of the inequalities:

$$
\boldsymbol{R}^{\prime}(\boldsymbol{Q}) \equiv \mathbf{B}_{r}^{\prime} \boldsymbol{Q}-\boldsymbol{b}_{r}=\mathbf{0}
$$

very similar to the form of the boundary conditions in Eq. (13). The unknown controls might also be limited:

$$
\boldsymbol{L}^{\prime}(\boldsymbol{C}) \equiv \mathbf{B}_{\ell}^{\prime} \boldsymbol{C}-\boldsymbol{b}_{\ell}=\mathbf{0} .
$$

The set of active restrictions is iteratively updated, until a feasible solution is obtained. The correctness of an introduced restriction can be checked from the sign of a corresponding Lagrange multiplier in the solution. A fully automatic procedure to ensure convergence to the restricted optimum is not believed possible, but reasonably efficient and reliable procedures are existing.

\subsection{Cost functions}

A control cost was in the present work defined as:

$$
\Pi_{c} \equiv \frac{1}{2} \int_{0}^{\mathrm{T}}\left(\sum_{i}\left(c_{i}(t)\right)^{2}\right) d t=\sum_{i} \sum_{j} \Pi_{i}^{j}=\boldsymbol{C}^{\mathrm{T}} \mathbb{C}_{c} \boldsymbol{C}
$$

with simple form for the matrix $\mathbb{C}_{c},[2]$.

Introducing an optimization of the dynamic movement with respect to maximum smoothness of movement, a 'cost' for jerkiness can be formulated, based on the idea in [4]. An expression for the integrated sum of squared jerk components:

$$
\Pi_{q} \equiv \frac{1}{2} \int_{0}^{\mathrm{T}}\left(\sum_{i}\left(j_{i}(t)\right)^{2}\right) d t=\boldsymbol{Q}^{\mathrm{T}} \mathbb{C}_{q} \boldsymbol{Q}
$$

can be obtained through the temporal interpolation, Eq. (8). Similarly, a cost for accelerations - or even for the velocities — can be introduced through other matrices $\mathbb{C}_{q}$ operating on the displacement component vector $\boldsymbol{Q}$.

\subsection{Discretized systems}

The algebraic non-linear equilibrium equations constitute the basis for all dynamic problems. The three classes of dynamic situations discussed in [1] all result in a non-linear set of equations, with the total and differential forms:

$$
\boldsymbol{F}(\boldsymbol{z})=\mathbf{0} \quad ; \quad d \boldsymbol{F}=\nabla \boldsymbol{F}(\boldsymbol{z}) d \boldsymbol{z}
$$

The solution vector $z$ has different meanings and contents. The displacement components, the control force values and the Lagrange multipliers are obtained through a Newton procedure. 


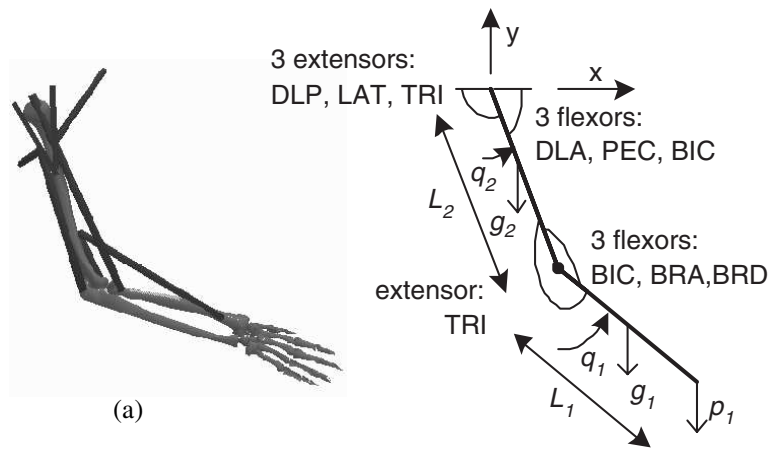

(b)

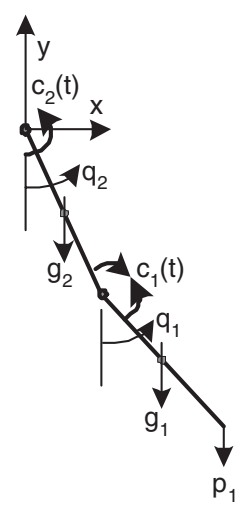

(c)

Figure 1: Problem definition: a) musculoskeletal system; b) measures, coordinates, loads, and muscles; c) resultant control moment at joints. Muscle notation as in Table 1.

An optimal path problem is formulated from a minimization of a scalar function. Allowing any of the cost functions above, the objective function is written:

$$
\begin{aligned}
\Pi\left(\boldsymbol{Q}, \boldsymbol{C}, \boldsymbol{\Lambda}_{E}, \boldsymbol{\Lambda}_{B}, \boldsymbol{\Lambda}_{r}, \boldsymbol{\Lambda}_{\ell}\right) & =\alpha_{c} \boldsymbol{C}^{\mathrm{T}} \mathbb{C}_{\mathbf{c}} \boldsymbol{C}+\alpha_{q} \boldsymbol{Q}^{\mathrm{T}} \mathbb{C}_{\mathbf{q}} \boldsymbol{Q} \\
& +\boldsymbol{\Lambda}_{E}^{\mathrm{T}} \boldsymbol{E}(\boldsymbol{Q}, \boldsymbol{C}, \boldsymbol{P})+\boldsymbol{\Lambda}_{B}^{\mathrm{T}} \boldsymbol{B}(\boldsymbol{Q}) \\
& +\boldsymbol{\Lambda}_{r}^{\mathrm{T}} \boldsymbol{R}^{\prime}(\boldsymbol{Q})+\boldsymbol{\Lambda}_{\ell}^{\mathrm{T}} \boldsymbol{L}^{\prime}(\boldsymbol{C})
\end{aligned}
$$

where $\alpha_{c}$ and $\alpha_{q}$ can be used to choose criterion for the minimization, and the Lagrange multipliers $\left(\boldsymbol{\Lambda}_{E}, \boldsymbol{\Lambda}_{B}, \boldsymbol{\Lambda}_{r}, \boldsymbol{\Lambda}_{\ell}\right)$ enforce the equilibrium and boundary equations, but also the current restrictions.

The optimal solution demands stationarity with respect to all variables in:

$$
\boldsymbol{z}=\left(\boldsymbol{Q}^{\mathrm{T}} \boldsymbol{C}^{\mathrm{T}} \boldsymbol{\Lambda}_{E}^{\mathrm{T}} \boldsymbol{\Lambda}_{B}^{\mathrm{T}} \boldsymbol{\Lambda}_{r}^{\mathrm{T}} \boldsymbol{\Lambda}_{\ell}^{\mathrm{T}}\right)^{\mathrm{T}}
$$

An algorithm for this was formulated in Matlab,[14].

\section{Numerical example: sagittal model of human arm}

A simplified sagittal two-link model of a human arm is analysed in $[2,15]$. The model, shown in Fig. 1 was used for a study of static and dynamic equilibrium. The data for the model were here chosen as $L_{1}=L_{2}=0.4 \mathrm{~m}, g_{1}=20 \mathrm{~N}$, $g_{2}=30 \mathrm{~N}, p_{1}=50 \mathrm{~N}$. The load multiplier was in this case assumed to affect self-weight, as well as applied load.

The muscle data in the model were collected from [16-19], Table 1 . The force capacities of all muscles were approximated as the physiological cross sectional 
Table 1: Used muscular data for distribution of moments. Moment arm MA1 is at elbow and MA2 at shoulder, positive for flexor, negative for extensor. Muscular area PCSA is physiological cross sectional area. Data collected from [16-19], cf. [15]. The abbreviated muscle names refer to: BIC, Biceps Brachii; BRD, Brachioradialis; BRA, Brachialis; TRI, Triceps brachii; DLP, Deltoideus (posterior part); DLA, Deltoideus (anterior part); PEC, Pectoralis major, clavicular part; LAT, Latissimus dorsi.

\begin{tabular}{|c|c|c|c|c|c|c|c|c|c|}
\hline & BIC & BRA & BRD & TRI & DLP & DLA & PEC & LAT & \\
\hline MA1 & 47.1 & 27.8 & 54.4 & -19.2 & 0 & 0 & 0 & 0 & {$\left[10^{-3} \mathrm{~m}\right]$} \\
MA2 & 15 & 0 & 0 & -34 & -33 & 21 & 11 & -43 & {$\left[10^{-3} \mathrm{~m}\right]$} \\
PCSA & 5.1 & 5.4 & 1.2 & 14.9 & 16.6 & 8.1 & 8.7 & 8.5 & {$\left[10^{-4} \mathrm{~m}^{2}\right]$} \\
\hline
\end{tabular}

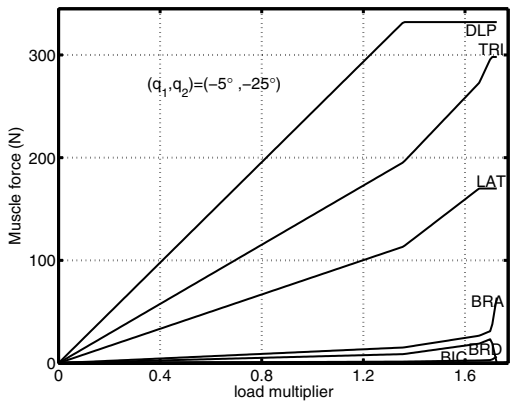

(a)

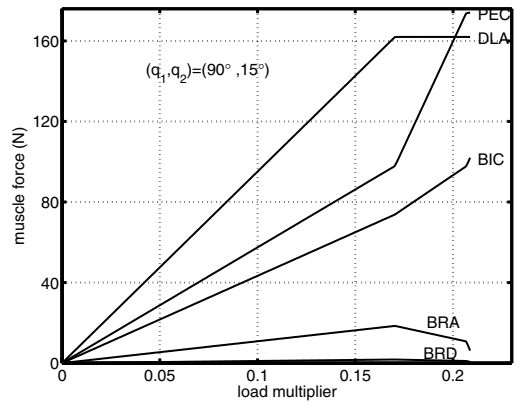

(b)

Figure 2: Variation of muscular forces for different load multipliers, in different configurations: a) posterior case $\left(q_{1}, q_{2}\right)=\left(-5^{\circ},-25^{\circ}\right)$; b) anterior case $\left(q_{1}, q_{2}\right)=\left(90^{\circ}, 15^{\circ}\right)$. Muscle notation as in Table 1.

areas ('PCSA') multiplied by a maximum nominal stress value of $200 \mathrm{kPa}$. More sophisticated expressions could be easily introduced.

Static equilibrium solutions were obtained for $-45^{\circ} \leq q_{2} \leq 155^{\circ}, q_{2}-10^{\circ} \leq$ $q_{1} \leq q_{2}+160^{\circ}$ in $10^{\circ}$ steps for each angle, and evaluated to give minimum summed squares of stresses in the redundant muscular system. Introducing the individual force capacities, the quasi-static muscular forces depend on the load multiplier (Fig. 2). The obtained system capacity can be shown as a function of the position of the wrist (Fig. 3).

The same arm model was studied in the dynamic range, solving for an optimal movement between an initial and a target state, minimizing the control force cost, Eq. (17), for the muscular moments $\left(c_{1}, c_{2}\right)$ in Fig. 1(c). A movement from a 


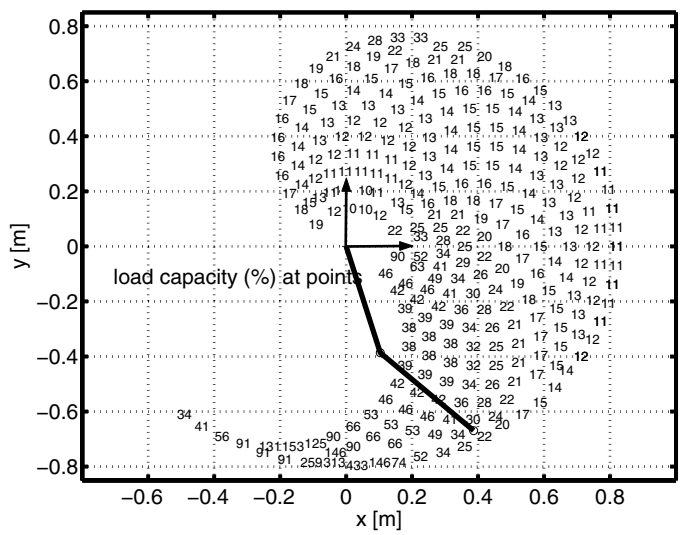

Figure 3: Load carrying capacity, expressed as a multiplier (\%) for the load, as function of the wrist position.

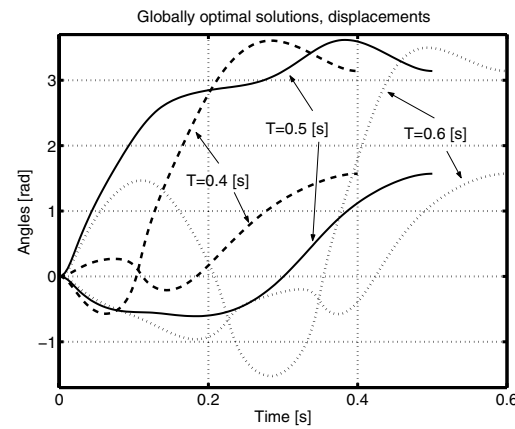

(a)

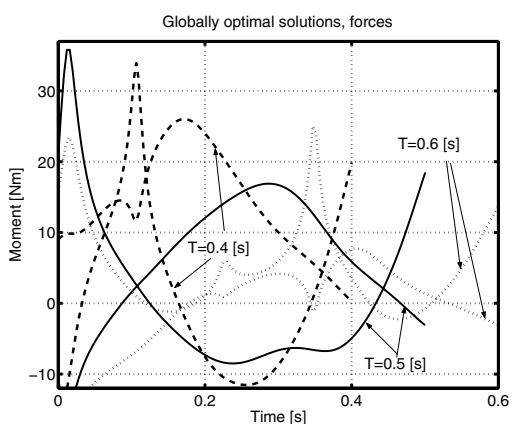

(b)

Figure 4: Globally optimal solutions, evaluated with $\left(N_{t}, N_{k}\right)=(128,129)$ for $T=0.4,0.5,0.6 \mathrm{~s}$ : a) displacements, angles; b) forces, moments.

hanging vertical configuration $\left(q_{1}, q_{2}\right)=(0,0)$ to a configuration with $\left(q_{1}, q_{2}\right)=$ $\left(\pi, \frac{\pi}{2}\right)$ was studied; both end configurations were given zero velocities, but accelerations were not specified. The problem shows several solutions, which are local optima for control force cost. For a time interval $T=0.5 \mathrm{~s}$, the global optimum corresponds to a rather smooth transition between initial and target state, (Fig. 4).

The same figure shows that the globally optimal solutions for $T=0.4 \mathrm{~s}$ and $T=0.6 \mathrm{~s}$ utilize pendulum type behaviors. Although the shown solutions are mathematically optimal under stated assumptions, all three solutions are physiologically impossible. For the case $T=0.5 \mathrm{~s}$, a restriction was therefore introduced $q_{2} \leq q_{1} \leq q_{2}+2.7$ rad., rather arbitrarily chosen. For this solution, the 


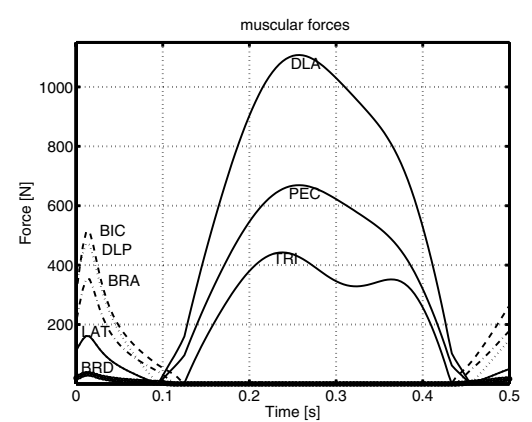

(a)

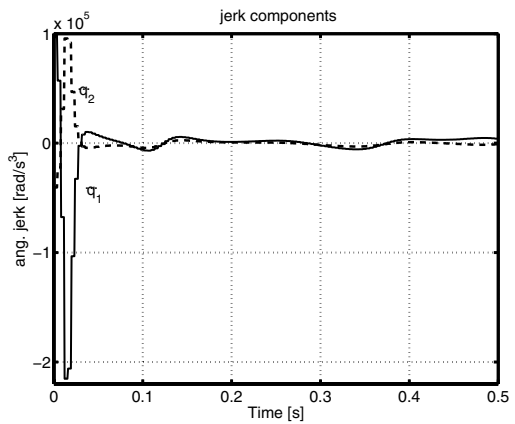

(b)

Figure 5: Post-processed results for the case $T=0.5 \mathrm{~s}$ in Fig. 4: a) muscular forces; b) evaluated jerks in the two displacement coordinates. Muscle notation as in Table 1 .

final weighted average control force norm was about $26 \%$ higher than in the unrestricted solution in Fig. 4, [2].

The optimized control moments can be distributed on the involved muscles, by the equivalent to a static optimization at each time instance, $[2,15]$. Using for this stage a criterion of minimum summed squares of muscular nominal stresses, the obtained muscular forces are shown in Fig. 5(a) for $T=0.5 \mathrm{~s}$ in Fig. 4. It is noted that the obtained forces are not necessarily the ones that would have been obtained, if the equilibrium equations had been formulated with these stresses as unknown controls.

Also the jerk components were evaluated. These are shown in Fig. 5(b) for the case $T=0.5 \mathrm{~s}$. Obviously, the solution based on minimal force cost gives a very jerky motion, which is probably far from a minimal-jerk solution.

\section{Concluding remarks}

The present paper has discussed a temporal finite element approximation for the dynamic behavior in structures and mechanisms, with a special interest in problems with low to moderate numbers of displacement coordinates. A basic methodology for solution of a wide set of dynamic simulations is thereby obtained. This gives a basis for further development in the directions of increasing bio-fidelity, but also towards an improved simulation efficiency. 


\section{References}

[1] Eriksson, A., Temporal finite element descriptions in structural dynamics. Proc. 7th Int. Conf. Comp. Struct. Techn., eds. B.H.V. Topping \& C.A. Mota Soares, Civil-Comp Press: Stirling, U.K., 2004.

[2] Eriksson, A., Optimal path simulations with temporal finite elements. $J$ Biomech, 2005. (submitted).

[3] Dahlquist, G. \& Björk, Å., Numerical Methods. Prentice-Hall: Engelwood Cliffs, 1974.

[4] Flash, T. \& Hogan, N., The coordination of arm movements: an experimentally confirmed mathematical model. J Neurosci, 5, pp. 1688-1703, 1985.

[5] Krebs, H., Aisen, M., Volpe, B. \& Hogan, N., Quantization of continuous arm movements in humans with brain injury. Proc Natl Acad Sci USA, 96, pp. 4645-4649, 1999.

[6] Okadome, T. \& Honda, M., Kinematic construction of the trajectory of sequential arm movements. Biol Cybern, 80, pp. 157-169, 1999.

[7] Piazzi, A. \& Visioli, A., Global mimimum-jerk trajectory planning of robot manipulators. IEEE Trans Ind Electr, 47, pp. 140-149, 2000.

[8] Secco, E.L., Visioli, A. \& Magenes, G., Minimum jerk motion planning for a prosthetic finger. J Robotic systems, 21, pp. 361-368, 2004.

[9] Phanindra, T. \& Majumdar, S., Selection of the cost function for determination of muscle forces. Proc. 7th Int. Conf. Comp. Struct. Techn., eds. B.H.V. Topping \& C.A. Mota Soares, Civil-Comp Press: Stirling, U.K., 2004.

[10] Eriksson, A., Temporal finite elements for target control dynamics of mechanisms. Comp Struct, 2004. (submitted).

[11] Géradin, M. \& Rixen, D., Mechanical vibrations. Theory and application to structural dynamics. Wiley: Chichester, 2nd edition, 1997.

[12] Zienkiewicz, O.C. \& Taylor, R.L., The Finite Element Method. Volume 1: The basis. Butterworth-Heinemann: Oxford, 5th edition, 2000.

[13] Cook, R.D., Malkus, D.S., Plesha, M.E. \& Witt, R.J., Concepts and Applications of Finite Element Analysis. Wiley, 4th edition, 2002.

[14] MathWorks, Inc., Matlab. Http://www.mathworks.com (June 2004), Version 6.1 .

[15] Heintz, S., Gutierrez, E. \& Eriksson, A., Static optimization for redundant force-limited muscle systems. J Biomech, 2004. (submitted).

[16] Lemay, M.A. \& Crago, P.E., A dynamic model for simulating movements of the elbow, forearm, and wrist. J Biomech, 29, pp. 1319-1330, 1996.

[17] Murray, W.M., Buchanan, T.S. \& Delp, S.L., The isometric functional capacity of muscles that cross the elbow. J Biomech, 33, pp. 943-952, 2000.

[18] Laursen, B., Jensen, B.R., Németh, G. \& Sjøgaard, G., A model predicting individual shoulder muscle forces based on relationship between electromyographic and 3D external forces in static position. J Biomech, 31, pp. 731-739, 1998.

[19] van der Helm, F.C.T., A finite element musculoskeletal model of the shoulder mechanism. J Biomech, 27, pp. 551-569, 1994. 